\title{
Promoviendo capacidades para la investigación: un esfuerzo en cadena
}

L

a creación de la Dirección de Investigación (DIRINV) en la Universidad Centroamericana, en 2001, respondió a la necesidad de potenciar la calidad y el impacto de las investigaciones que se realizan en la universidad. Desde entonces, la oficina ha venido consolidando una estrategia nacida principalmente de su contacto directo con profesores y estudiantes, de su ejecución de proyectos específicos financiados por la cooperación internacional, de su experiencia editorial como responsable de UCA Publicaciones, y de su vinculación con los esfuerzos comunes de toda la universidad, plasmados en el Plan Estratégico. A lo largo de trece años, la oficina ha podido diseñar, poner en práctica y evaluar, distintas iniciativas, lo que nos ha permitido ganar experiencia y perspectiva sobre los verdaderos retos que se deben enfrentar para hacer investigación en nuestro contexto.

Por un lado, hemos podido constatar que nuestros investigadores tienen - y aprovechan - la clara ventaja de conocer de primera mano los fenómenos que los investigadores extranjeros enfrentan por primera vez cuando vienen a realizar sus trabajos de campo. Sin embargo, realizar investigación y publicación científica desde nuestro país implica vencer retos específicos de nuestra realidad. Un investigador de la UCA tiene dificultades que investigadores de otros países no suelen tener. Por ejemplo, todavía se queda corto nuestro acceso a libros y revistas científicas, hace falta capacidad de escritura y lectura, y aún surgen muchas investigaciones que carecen de solidez conceptual y penetración analítica. Con estos problemas en mente, los esfuerzos de la DIRINV para promover la investigación en la UCA se concretan en iniciativas de cuatro tipos: 1. la inclusión de la investigación en el sistema de incentivos para los profesores de la universidad; 2. la promoción de los estudios de doctorado; 3. la dotación de fondos a equipos interdisciplinarios; y 4. la promoción de capacidades básicas para la investigación y la publicación.

En concreto, las iniciativas con las que la DIRINV ha iniciado el año 2014 son las siguientes:

- La convocatoria a tres fondos de US\$14,000 para financiar tres proyectos, cada uno en una de las siguientes líneas de la Agenda de Investigación de la UCA: Estado, democracia y ciudadanía; Cambio climático y gestión ambiental; Educación, ética y cultura ciudadana.

- Dos cursos para redacción de artículos científicos: uno para profesores de ciencias sociales y afines, y otro para profesores de ciencias naturales y afines.

- El VIII Congreso de Investigación, en el que se presentará la ponencia magistral: "La contribución y desafíos de la investigación universitaria ante las problemáticas de la violencia y la impunidad", a cargo de la investigadora Leticia Salomón de la Universidad Nacional Autónoma de Honduras.

- La convocatoria para el XIV Encuentro de Jóvenes Investigadores.

Estas iniciativas no deben verse como actividades separadas cuyos efectos se limitan a lo expresado en sus objetivos específicos. Son parte de una cadena de esfuerzos; ni una sola de ellas puede, por sí sola, tener un impacto determinante o, en su defecto, ser un fracaso o desperdicio. Ni los fondos por sí solos, ni los cursos cortos o doctorados, ni los congresos, pueden promover la investigación cuando las personas no están motivadas o genuinamente interesadas en la generación de conocimiento. Quizás un reto que la DIRINV deberá enfrentar a futuro es la cuestión de cómo promover la carrera del investigador, y mejorar la fortaleza teórica de la investigación y las publicaciones que realizamos. En nuestro medio es común ver que el mayor énfasis y esfuerzo esté puesto en el desarrollo y aplicación de una metodología, que muchas veces está vacía. Quizás el siguiente eslabón en la cadena de esfuerzos sea insistir en la importancia de dedicarle tiempo a la lectura y la escritura. Esta visión deberá ser incluida en nuestro sistema de incentivos, promoviendo investigaciones que vayan más allá de la ejecución de un proceso metodológico.

Wendy Bellanger

Editora 